\title{
A second neutron star in M4?*
}

\author{
J. Kaluzny, A. Rozanska, M. Rozyczka, W. Krzeminski \\ Nicolaus Copernicus Astronomical Center, Bartycka 18, 00-716 Warsaw, Poland \\ and \\ Ian B. Thompson \\ Observatories of the Carnegie Institution of Washington, 813 Santa Barbara Street, \\ Pasadena, CA 91101
}

\begin{abstract}
We show that the optical counterpart of the X-ray source CX 1 in M4 is a $\sim 20$ th magnitude star, located in the color-magnitude diagram on (or very close to) the main sequence of the cluster, and exhibiting sinusoidal variations of the flux. We find the X-ray flux to be also periodically variable, with $\mathrm{X}$ ray and optical minima coinciding. Stability of the optical light curve, lack of UV-excess, and unrealistic mean density resulting from period-density relation for semidetached systems, speak against the original identification of CX 1 as a cataclysmic variable. We argue that the X-ray active component of this system is a neutron star (probably a millisecond pulsar).
\end{abstract}

Subject headings: binaries: close - globular clusters: general - globular clusters: individual (M4) - cataclysmic variables - X-rays: stars

\section{Introduction}

Globular clusters (GCs) contain a large population of exotic binary stars with degenerate components, and many of these are X-ray sources with various degrees of activity. Numerous such sources have been detected by the Chandra space observatory in over 80 distinct clusters, providing a valuable tracer population for large-scale numerical simulations of cluster evolution (Poolev 2010).

* Based on observations made with the NASA/ESA Hubble Space Telescope, and obtained from the Hubble Legacy Archive, which is a collaboration between the Space Telescope Science Institute (STScI/NASA), the Space Telescope European Coordinating Facility (ST-ECF/ESA) and the Canadian Astronomy Data Centre (CADC/NRC/CSA). 
One of the clusters surveyed by Chandra is M4 (NGC 6121). Eight of the X-ray sources found in its core region were classified as chromospherically active binaries, one as a cataclysmic variable $(\mathrm{CV})$, and one was identified with the millisecond pulsar PSR1620-26 (Bassa et al. 2004, hereafter BPH). The CV-candidate, referred to as CX 1 in BPH, is the brightest among them $\left(L_{0.5-2.5 \mathrm{keV}}=8.3 \times 10^{31} \mathrm{erg} \mathrm{s}^{-1}\right)$. BPH identified it with a bright $V_{555}=17.37$ star, located on the main sequence of M4 just below the turnoff. They argued that its X-ray luminosity is too high for a magnetically active binary composed of two mainsequence stars (i.e., a BY Dra system), while its optical luminosity is too low for an active binary with a subgiant (i.e., an RS CVn system). Their identification of CX 1 as a CV was additionally supported by its rather hard X-ray spectrum $\left(L_{0.5-1.5 \mathrm{keV}} / L_{1.5-6.0 \mathrm{keV}}=1.22\right)$, and a high ratio of $\mathrm{X}$-ray and optical fluxes.

$\mathrm{BPH}$ noted that the optical centroid of the bright star is offset by $2 \sigma$ from the Chandra position of CX 1, so the identification might be questionable. If that were the case, the actual counterpart of CX 1 could be much fainter, possibly lost in the glare of the brighter star. Indeed, the examination of the M4-photometry published by Anderson et al. (2008) showed that the object identified by $\mathrm{BPH}$ was a blend of two stars. In the present Letter we demonstrate that the X-ray activity is associated with the much fainter and redder component of the blend. We argue that CX 1 is a millisecond pulsar or qLMXB rather than a cataclysmic variable.

\section{Observations}

\subsection{Ground-based}

M4 was observed in nine seasons between 1995 and 2009 as one of the objects included in the CASE project (Kaluzny et al. 2005). Altogether, $1683 \mathrm{~V}$-band images were collected with the 2.5-m du Pont telescope at Las Campanas Observatory, using the same CCD camera and the same filter. Exposure times ranged from 20 s to $120 \mathrm{~s}$, with a median value of $45 \mathrm{~s}$. The median seeing was $0.98^{\prime \prime}$. The photometric analysis was based on a combination of image subtraction and profile photometry techniques. We used scripts employing the Daophot/Allstar package (Stetson 1987) and a modified version of the ISIS code (Alard \& Lupton 1998); see Kaluzny et al. (2010) for details. Using ISIS, one obtains a light curve in the form of differential counts. This allows for the photometry of variable objects even in those cases when they are tightly blended. Conversion of the light curve from differential counts to stellar magnitudes requires determination of the pedestal flux for a target star on the reference image (see Sect. 2.2). Further details concerning the observations and the calibration of the photometry will be given in a forthcoming paper (Kaluzny et al. 2012; in preparation). 
The optical counterpart of CX 1 proposed by BPH turned out to be a low-amplitude periodic variable with a stable light curve, suggestive of an ellipsoidal and/or irradiation effect. The analysis of variance, implemented in the Tatry code (Schwarzenberg-Czerny 1996), yielded a period $P_{1}=0.2628216(1) \mathrm{d}$. The linear ephemeris

$$
H J D_{\min }=2,454,656.7394(16)+E \times P_{1}
$$

provides a good fit to all data - we found no evidence for any change of period, amplitude or average magnitude from one observational season to another. There is, however, an ambiguity concerning the period: a value $P_{2}=0.5256432(1)$ d, i.e. two times larger than $P_{1}$, is also perfectly acceptable (see Fig. 1). We address this issue in Sects. 2.2 and 3 .

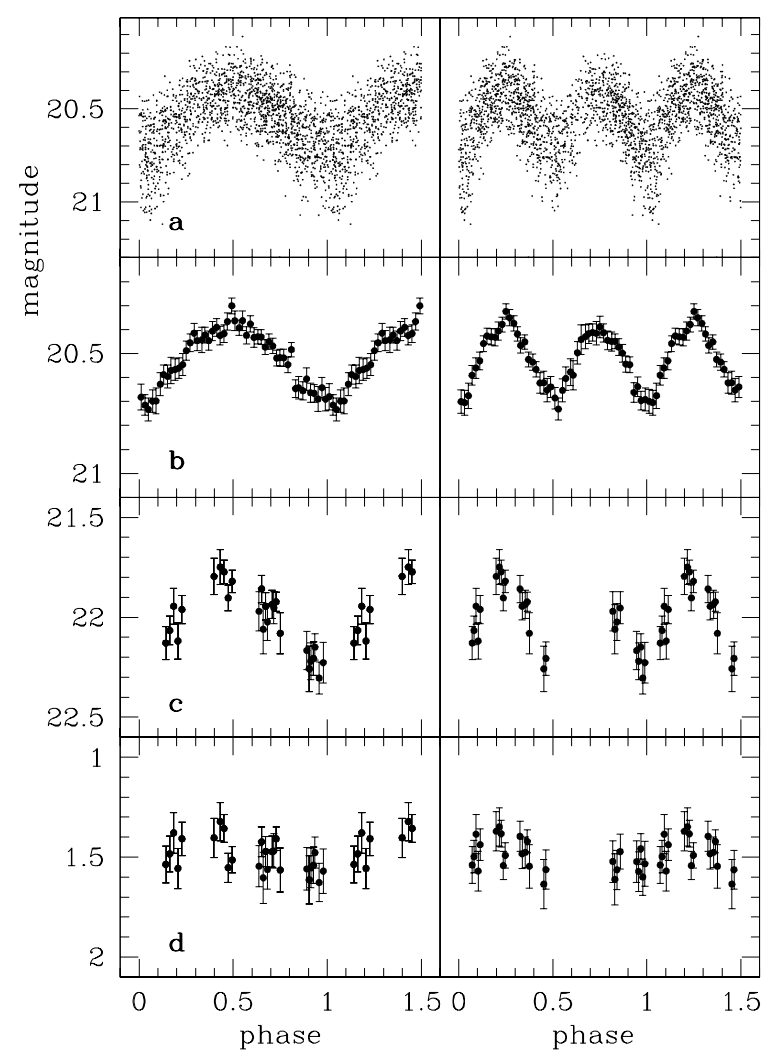

Fig. 1. - Left column: optical light curves of the star \#5274 phased with the ephemeris (1). a: ground-based, $V$-band (mean error of a single observation is $0.20 \mathrm{mag}$ ); b: the same data binned (50 bins per period); c: archival HST data, F435W-band, ACS/VEGA system; d: color index $(\mathrm{F} 435 \mathrm{~W}-V)$. Right column: the same data phased with $P_{2}$ used in eq. (1) instead of $P_{1}$. 


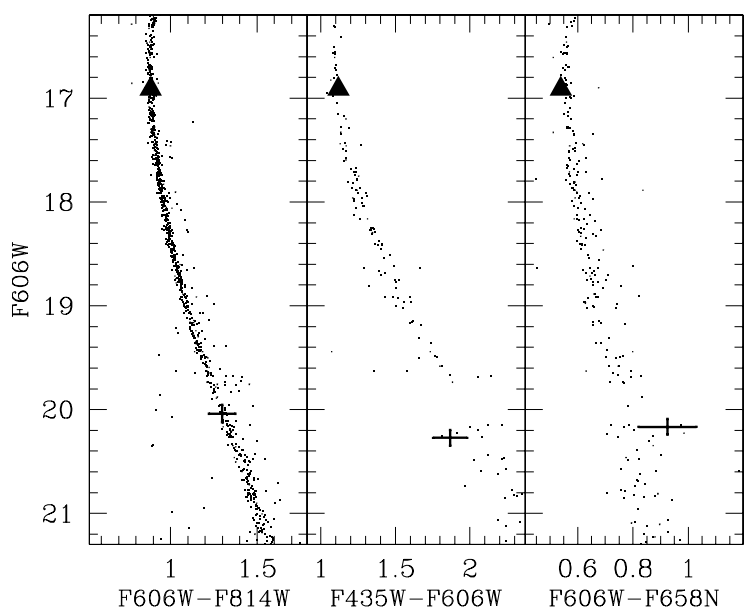

Fig. 2.- Location of stars \#5273 (triangle) and \#5274 (point with errorbars) on the CMD of M4. All magnitudes are given in the ACS/VEGA system. The F658N filter is centered on the $\mathrm{H} \alpha$ line; the $\mathrm{F} 606 \mathrm{~W}$ magnitudes are interpolated to the phases at which frames in filters F814W, F435W and F658N were taken (0.666, 0.513 and 0.073, respectively). The F606W-F814W CMD is taken directly from Anderson et al. (2008), and the remaining two CMDs are extracted from subfields of the images mentioned in Sect. 2.2.

\subsection{HST}

The optical counterpart of CX 1 is visible not only in $H S T / W F P C 2$ images analysed by $\mathrm{BPH}$, but also in several images obtained with $H S T / A C S$. A closer inspection of those data reveals that it is actually a pair of stars, separated by $0.15^{\prime \prime}$. These stars were resolved in the F606W/F814W photometry performed by Anderson et al. (2008), and placed in their data base with F606W, F814W, $V$ and $I$ magnitudes. According to Anderson et al. (2008), the brighter component of the pair (star \#5273) has $V=17.170$, while the fainter one (star \#5274) reaches only $V=20.650$. We note that the difference between $V=17.170$ of Anderson et al. (2008) and $V_{555}=17.37$ of $\mathrm{BPH}$ originates from different photometric systems used by those authors.

The HST archive contains four images taken with the F814W filter between 05:27 and 08:04 UT on 2006-03-05 with $T_{\exp }=40 \mathrm{~s}$ (Program ID 10775, PI A. Sarajedini). From them, we extracted the photometry of both components of the pair and checked which one was variable. The star \#5273 was constant to within observational errors (amounting to $0.015 \mathrm{mag}$ ), while \#5274 exhibited fluctuations with an amplitude of $\sim 0.5 \mathrm{mag}$. The final proof that the fainter component of the pair is responsible for the variability detected in the ground-based data came from the analysis of a yet another set of 30 archival HST/ACS images, collected with the F435W filter on 2006-04-08 (Program ID 10615, PI S. Anderson; 
all but three of these obtained with $T_{\exp }=340 \mathrm{~s}$ ). We extracted the photometry with Dophot/Allstar (Stetson 1987), and transformed it to the ACS-VEGA system, following the prescription of Sirianni et al. (2005). Again, the brighter star was remarkably constant $\left(\sigma_{\mathrm{F} 435 \mathrm{~W}}=0.013 \mathrm{mag}\right)$, showing no periodicity with $P_{1}$ or $P_{2}$ (in fact, it showed no periodicity whatsoever).

Based on $V$-magnitudes given by Anderson et al. (2008), we estimated the instrumental magnitude of the star \#5274 in our ground-based reference image used for image subtraction. This allowed us to transform the ISIS light curve from differential counts to magnitudes (the curve was extracted at the position of star \#5274, and the phase difference between ISIS template and ACS F606W image was accounted for). Next, we phased the $V$-band magnitudes of the star \#5274 using the ephemeris (11), averaged them using phase bins $\Delta \phi=0.02$, and interpolated them onto phases at which the F435W frames were taken. The interpolation enabled us to calculate the $(V-\mathrm{F} 435 \mathrm{~W})$ color as a function of phase. We found that the maxima of the light curve were bluer by $\sim 0.2$ mag than the minima (see Fig. 11). Such a behaviour is indicative of the irradiation effect, which produces a light curve with one maximum per orbital period, as is observed when $P_{1}$ is used phasing. Alternatively, $P_{2}$ produces two maxima per period - a feature characteristic of the ellipsoidal effect (however, in this case the irradiation may also play a role). In Sect. 3 we argue that the actual orbital period $P_{\text {orb }}$ is most probably equal to $P_{1}(\approx 0.2628 \mathrm{~d} \approx 6.3 \mathrm{~h})$.

\subsection{Chandra}

M4 was observed three times with the ACIS-S spectrometer on the board of Chandra X-ray Observatory on 2000 June 30 (Obs. ID 946), 2007 July 06 (Obs. ID 7447), and 2007 September 18 (Obs. ID 7446) for 7.17, 12.63 and $13.31 \mathrm{~h}$, respectively. During all observations the target was centered on the S3 chip, and the integration time was 3.24 sec per frame in the TIMED mode. All data were downloaded from the Chandra archive. The observations from 2000 June 30 were analyzed earlier by BPH, but we decided to repeat the analysis so that all data sets would be processed in exactly the same way. For the calibration of each observation the same version of CALDB 3.4.0 was employed.

CX1 was localized using the physical coordinates given by BPH (i.e. $\alpha=16^{\mathrm{h}} 23^{\mathrm{m}} 34.12^{\mathrm{s}}$ and $\delta=-26^{\circ} 31^{\prime} 34.85^{\prime \prime}$ ), and the light curves were exctracted using CIAO 4.3 software. All data sets were filtered for energies $0.5-7 \mathrm{keV}$, as suggested in CIAO ACIS analysis threads. Background flaring was accounted for with the help of CIAO tools dmextract and lc_clean. Since the background was practically not flaring, good-time intervals did not differ much from total exposure times, amounting to 6.6, 12.06, and $12.96 \mathrm{~h}$ for observations 946,7447 , 


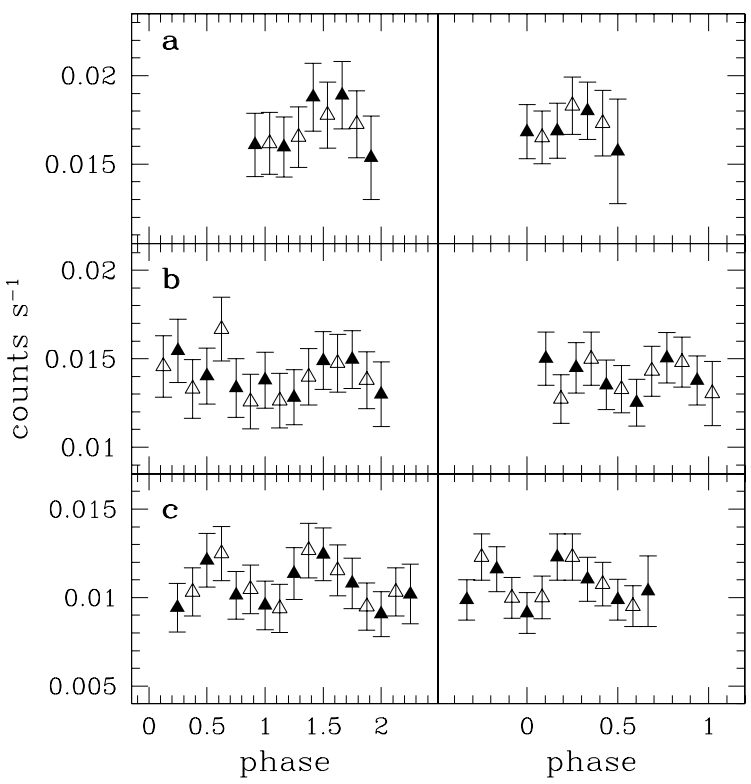

Fig. 3.- X-ray light curves of CX 1. Left column: counts phased with the epehmeris (1) without phase-folding, and binned in four intervals per period (filled triangles). Empty triangles: the same binning, but the bins are shifted by $\Delta \phi=0.125$. Right column: counts phased with $P_{2}$ used in eq. (1) instead of $P_{1}$, and binned in six intervals per period (filled triangles). Empty triangles: the same binning, but the bins are shifted by $\Delta \phi=0.083$. No phase-folding was applied. Panels a, b and c show observations from 2000-06-30, 2007-07-06 and 2007-09-18, respectively. $\phi=0$ corresponds to the minimum of the optical light curve.

and 7446, respectively.

The source region was defined as a circle with a radius of $3^{\prime \prime}$ centered on CX 1 , and the background was sampled in a surrounding annulus with inner and outer radius of $3^{\prime \prime}$ and $10^{\prime \prime}$ (we checked that varying the outer radius within reasonable limits did not change the shape of the extracted light curves). Data sets 946, 7447, and 7446 yielded, $410 \pm 20,610 \pm 24$, and $494 \pm 22$ counts, respectively. The counts were binned in intervals of 0.25 and 0.167 of the period for $P_{1}$ and $P_{2}$, respectively, and the resulting X-ray light curves are shown in Fig. 3. While rather noisy, they exhibit a modulation similar to that of the optical light curves, with a minimum near $\phi=0$.

The same regions were used for the extraction of spectra with the specextract tool. To within standard errors, in each data set the same number of counts was detected as before. The spectra were binned, and each bin contained at least 15 counts. XSPEC v.12.6.0 was used for fitting. We tried to fit three models: a power law, a black-body, and thermal bremsstrahlung, each convolved with a warm absorption model. For all three data sets the 


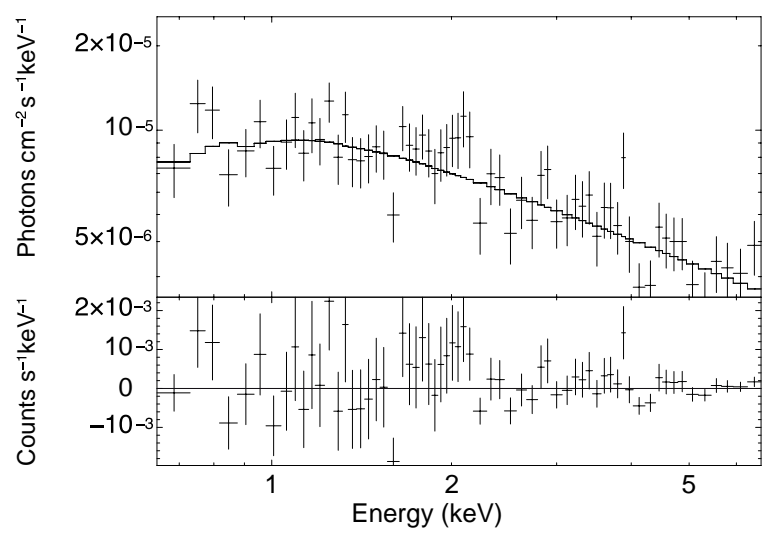

Fig. 4.- X-ray spectrum of CX 1, derived from the combined exposures described in the text. The lower panel shows the residual to the power law fit.

only reasonable fit was the power law. The black-body produced either $r \chi^{2} /$ dof $>2$, or warm absorption dropping to zero, whereas thermal bremstralung required an unacceptably high temperature $(199 \mathrm{keV})$.

The power law fit to the 946 data set yielded an absorbing column density $N_{H}=$ $2.29 \pm 1.04 \times 10^{21} \mathrm{~cm}^{-2}$, and photon index $\Gamma=0.96 \pm 0.17\left(\chi^{2} /\right.$ dof $\left.=25.5 / 22=1.16\right)$, which agrees well with the best-fit results of BPH, who obtained $N_{H}=(1.95-2.86) \times 10^{21} \mathrm{~cm}^{-2}$ and $\Gamma=0.99 \pm 0.17\left(\chi^{2} /\right.$ dof $\left.=33.3 / 39=0.85\right)$. The absorbing column density derived from fitting also agrees with $N_{H}=2.18 \times 10^{21}$, converted from the visual extinction $A_{V}=1.22$ (Kaluzny et al. 2012, in preparation) according to the prescription of Predehl \& Schmitt (1995). Assuming a distance to M4 of $2.2 \mathrm{kpc}$ (Harris 1996), we derived an unabsorbed X-ray luminosity $L_{0.5-2.5 \mathrm{keV}}=3.4 \times 10^{31} \mathrm{erg} / \mathrm{s}$, and a hardness ratio $L_{0.5-1.5 \mathrm{keV}} / L_{1.5-6.0 \mathrm{keV}}=0.21$. Similar values were obtained for the remaining two observations.

To improve the photon statistics, we combined all spectral data using the CIAO tool combine_spectra. The resulting cumulative spectrum of 1520 counts was binned in such a way that each bin contained at least 20 counts. The best-fitting model was again a power law convolved with warm absorption (Fig. (4), and its parameters did not differ much from those characterizing the individual spectra. We obtained $N_{H}=1.71 \pm 0.4 \times 10^{21} \mathrm{~cm}^{-2}$, and $\Gamma=0.87 \pm 0.08\left(\chi^{2} /\right.$ dof $\left.=67.84 / 61=1.11\right)$, with $L_{0.5-2.5 \mathrm{keV}}$ and $L_{0.5-1.5 \mathrm{keV}} / L_{1.5-6.0 \mathrm{keV}}$ amounting to $2.66 \times 10^{31} \mathrm{erg} / \mathrm{s}$ and 0.19 , respectively

Our fit is significantly harder than that of $\mathrm{BPH}$, resulting in a smaller hardness ratio. Their ratio is close to unity, but it was computed using correction factors averaged over three fits, whereas ours is derived from the best fit to the combined spectrum obtained from 
a much larger number of counts.

\section{Discussion and conclusions}

According to Zloczewski (2012), the star \#5273 is a proper-motion member of M4. Upon averaging the subtracted ISIS-images on a seasonal basis, we found no evidence of bipolar residuals at the position of its weak companion. Such residuals are observed for objects with noticeable proper motions with respect to the surrounding stellar field (Eyer \& Wozniak 2001). Given the time base and the pixel scale of our ground-based images, we rule out a relative proper motion of the star \#5274 in excess of 10 mas/y. Since on the propermotion diagram most of the field stars are separated from the cluster members by 15 mas/y (Zloczewski 2012), this is consistent with the star \#5274 being a member of M4.

BPH tentatively classified CX 1 as a CV. However, between 1995 and 2008 average optical luminosity and amplitude of the star \#5274 remained constant to better than 0.05 mag. This would be very unusual for a $\mathrm{CV}$ whose substantial X-ray emission implies active accretion via $\mathrm{L} 1$ overflow. If CX 1 is indeed such a system, despite its stability, then its visible component should be a main-sequence star filling its Roche lobe, whose average density can be calculated from the formula

$$
\bar{\rho}\left[\mathrm{g} \mathrm{cm}^{-3}\right]=107 P_{\text {orb }}^{-2}[h],
$$

accurate to $3 \%$ for mass ratios $0.01<q<1.0$ (Eggleton 1983$)$. For $P_{1}(\approx 0.2628 \mathrm{~d})$ and $P_{2}(\approx 0.5256 \mathrm{~d})$ we get a $\bar{\rho}$ of 2.8 and $0.7 \mathrm{~g} \mathrm{~cm}^{-3}$, respectively. Since an $11 \mathrm{Gyr}$ old mainsequence star with $M_{\mathrm{V}}=7.15$ and $[\mathrm{Fe} / \mathrm{H}]=-0.7$ should have $\bar{\rho}=4.04 \mathrm{~g} \mathrm{~cm}^{-3}$ (Dotter et al. 2008), the orbital period of CX 1 cannot be equal to $P_{2}$. $P_{1}$ might be considered marginally acceptable, were it not for the extraordinary stability of the star \#5274.

Another argument against CX 1 being a cataclysmic variable follows from the groundbased UBV photometry of M4 published by Mochejska et al. (2002). Between the outbursts, cataclysmic variables typically have $(U-V) \approx-1$ (Warner 1995). In the data of Mochejska et al. (2002) the stars \#5273 and \#5274 are blended, and the $(U-V)$ index of the unresolved blend amounts to 1.016, locating it right in the middle of the upper main sequence in the $V / U-V$ diagram. With $(U-V)=-1(-0.6)$, the star \#5274 would have an $U$ of $19.55(19.95) \mathrm{mag}$, and the color of the blend would be shifted $\delta(U-V)=-0.28$ $(-0.20)$. Such shifts would be easily seen on the $V /(U-V)$ diagram of (Mocheiska et al. 2002).

We conclude that mean density, stability and color arguments taken together make the interpretation of CX 1 as a cataclysmic variable very unlikely. The only viable alternative is a 
system whose primary is a neutron star instead of a white dwarf. If $P_{\text {orb }}=P_{1}$ then the optical variability of CX 1 must originate predominantly from irradiation (cf. Sect 2.2). However, even in this case the star \#5274 is likely to nearly fill its Roche lobe (first - it is heated, and therefore swollen; second - there must be a Roche-lobe overflow for the accretion to go on, and the X-rays to be produced). Alternatively, if $P_{\text {orb }}=P_{2}$ then the ellipsoidal effect becomes the main cause of optical variations. In any case, the semi-detached configuration should be a reasonable approximation of the CX 1 system, and the estimates of the mean density of the star \#5274 based on equation (2) remain valid. Having the same luminosity and temperature as similarly located main-sequence stars of M4, the star \#5274 must also have the same radius $R \approx 0.6 R_{\odot}$ (Dotter et al. 2008). For that size one gets a mass of $\sim 0.4 M_{\odot}$ and $\sim 0.1 M_{\odot}$, respectively for $P_{1}$ and $P_{2}$. Since the corresponding main-sequence mass is $0.58 M_{\odot}$, the Roche-lobe filling companion of CX 1 would have to be ablated either slightly (if $P_{\text {orb }}=P_{1}$ ) or very strongly (if $P_{\text {orb }}=P_{2}$ ). Simple experiments with the PHOEBE tool (Prša \& Zwitter 2005) show that the $V$-light curve for $P_{1}$ can be easily recovered, e.g. in a system with neutron star of $1.5 M_{\odot}$, semimajor axis of $2.3 R_{\odot}$, inclination of $\sim 20^{\circ}$ and illuminated-area temperature $20 \%$ higher than the rest of the photosphere (obviously, this does not prove that $P_{1}$ is the actual orbital period, nor the semi-detached configuration is the only possible).

For $P_{\text {orb }}=P_{1}$ there is only one X-ray minimum per period, which can be naturally explained as an eclipse, reminiscent of those observed in systems containing millisecond pulsars J0024-7203W in 47 Tuc or J1740-5340A in NGC 6397 (Heinke 2011). Two X-ray mimima per period, appearing for $P_{\text {orb }}=P_{2}$ (see Fig. 3), would be very hard to explain. We conclude that, while $P_{2}$ cannot be ruled out, it seems much less likely than $P_{1}$.

Thus, the available data favor the possibility that CX 1 is composed of a neutron star accompanied by a $\sim 0.6 M_{\odot}$ main-sequence star or a partially ablated, slightly oversized $\sim 0.4 M_{\odot}$ star. If this interpretation is correct, then it must be a millisecond pulsar or a qLMXB. The X-ray luminosity and hardness ratio derived in Sect. 2.3 support the first possibility; in fact, they locate CX 1 among the brightest millisecond pulsars shown in Fig. 6 of Bogdanov et al. (2010) (the nondetection of the radio emission may be due to an unfavourable orientation). As for the radiation mechanism, the strongly nonthermal spectrum suggests that the dominant source of X-rays may be a relativistic shock, produced by the pulsar wind interacting with the matter from the optical companion (Bogdanov et al. 2005). Future observations with XMM-Newton (more detailed X-ray light curves), HST (multicolor photometry) and VLT or Gemini South (IR spectroscopy with adaptive optics) should resolve the period ambiguity and more precisely establish the nature of this interesting object. 
This research has made use of the software provided by the Chandra X-Ray Center in the application package CIAO. JK and MR were supported by the grant N N203 379936 from the Polish Ministry of Science and Higher Education. We thank the anonymous referee for helpful comments, and in particular for noting an error in the phasing of the F435 light curve.

Facilities: HST, CXO

\section{REFERENCES}

Alard, C., Lupton, R. H 1998, ApJ, 503, 325

Anderson, J. et al. 2008, AJ, 135, 2055

Bassa, C. et al. 2004, ApJ, 609, 755

Bogdanov, S. et al. 2010, ApJ, 709, 241

Bogdanov, S., Grindlay, J. E., \& van den Berg, M. 2005, ApJ, 630, 1029

Dotter, A. et al. 2008, ApJS, 178, 89

Eggleton, P. P. 1983, ApJ, 268, 368

Eyer, L., \& Woźniak, P. R 2001, MNRAS, 327, 601

Harris, W. E. 1996, AJ, 112, 1487 (www.physics.mcmaster.ca/Globular.html)

Heinke, C. O. 2011, arXiv:1101.5356

Kaluzny, J. et al. 2005, AIP Conf. Proc., Vol. 752, "Stellar Astrophysics with the World's Largest Telescopes", ed. J. Mikolajewska and A. Olech, p. 70

Kaluzny, J., Thompson, I. B., Krzeminski, W., Zloczewski, K. 2010, Acta Astron., 60, 245

Mochejska, B., Kaluzny, J., Thompson, I., \& Pych, W. 2002, AJ, 124, 1486

Pooley, D. 2010, Proc. Natl. Acad. Sci., 107, 7164

Predehl, P., \& Schmitt, J. H. M. M. 1995, A\&A, 293, 889

Prša, A., \& Zwitter, T. 2005, AJ, 628, 426 
Schwarzenberg-Czerny A. 1996 ApJ, 460, L107

Sirianni, M. et al. 2005, PASP, 117, 1049

Stetson, P. B. 1987, PASP, 99, 191

Warner, B. 1995, Cataclysmic Variable Stars (Cambridge: Cambridge University Press)

Zloczewski, K. 2012, PhD thesis, NCAC 